\title{
A Study of Spontaneous Sweating of Ring and Little Fingers in Leprosy \\ (A Preliminary Communication)
}

\author{
DR B. B. GOKHALE, м.в.в.s., D.v.D. and MR S. G. PANSE \\ Department of Dermatology, Sassoon Hospitals, Poona, India
}

The volar surface of human hands sweats spontaneously and perpetually. The sweating rate is under the control of autonomic nerves and is considered a fairly reliable indicator of the ability of the human volar sweat glands to respond to sudorific stimulii if the sweat glands are normal. Therefore it can be usefully employed in the assessment of extent of involvement of autonomic innervation of the part supplied. The sweating rate can be easily measured simply by counting the number of sweat spots.

Anidrosis is a common feature of leprosy. Disturbances in sweating in leprosy are attributed to affection of the audorific nerves and the sweat glands themselves. The ulnar (a mixed nerve) is frequently affected in the disease, producing alterations, both in the somatic and autonomic activity in the area supplied. Consequently changes are noted in the sweating rate of the ring and little fingers and the hypothenar eminences of the hands.

Any test employed in leprosy work should be such that it could be easily performed in the field by paramedical personnel, without much instrumentation. It should be quantitative, simple and not very time-consuming; so that the patients would easily return for re-examination from time to time. With these objectives in mind the Wada ${ }^{2}$ (1948) test for detection of secretion of sweat as modified by Silver $A^{1}$ ( 1963 ) was employed in this study of secretory activity of the volar sweat glands of ring or little fingers of subjects who have leprosy.

\section{METHOD}

The area under study was clean dried with absolute alcohol. A circular area was marked on the volar aspects of the terminal phalanges of ring and little fingers. A cap of a thermometer cover was used to mark a circle. It encircled an area of I 5 o square $\mathrm{mm}$. The sweat pores were counted by using a magnifying lens with a built-in illuminating bulb of a dry cell torch. It is necessary to test identical contra-lateral sites as well as the identical areas in different subjects to reduce the effect of such factors as variation in the population density of sweat glands, the number of nerve endings, and callosities etc. The counting was done 15 minutes after the application of iodine and a thin layer of starch on to the area under examination. Under this magnification they appeared as circular black spots arranged along the epidermal ridges.

\section{A T E R I A L}

In this work, we have studied hands of healthy subjects (Group A) along with those of patients subject to leprosy without any obvious deformity due to involvement of the motor element of the ulnar nerve (Group B) and hands in leprosy patients with obvious deformities (Group C) due to affection of the motor component of the ulnar nerve irrespective of the type of disease. In these patients we recorded the type of leprosy along with other relevant clinical data.

Readings were taken in each case on three successive days during the same hours of the day (8.oo a.m. to 9.30 a.m.) in a quiet room. To avoid excitement the patients were made to lie comfortably for 30 minutes in the experimentation room prior to taking actual readings. All the readings of all the patients were made in the lying down position. As there was no significant variability within the set of the three readings taken on successive days in any of the cases (statistically tested), a mean of the three readings was used in all the cases for further calculations.

Some subjects were studied every month all round the year to observe any seasonal variations in sweating rate.

The study of healthy hands all round the year indicates that there are no significant variations 
in the sweating rate under the ordinary conditions of seasonal changes of temperature and humidity. It has become apparent that relatively extreme thermal stimulii are required to activate simultaneously one and all the functional sweat glands in any given area.

RESULTS

As it was statistically found out that the groups $\mathrm{A}, \mathrm{B}$ and $\mathrm{C}$ differed from each other in respect of their readings (Analysis of variants table was used) the results have been recorded as follows:

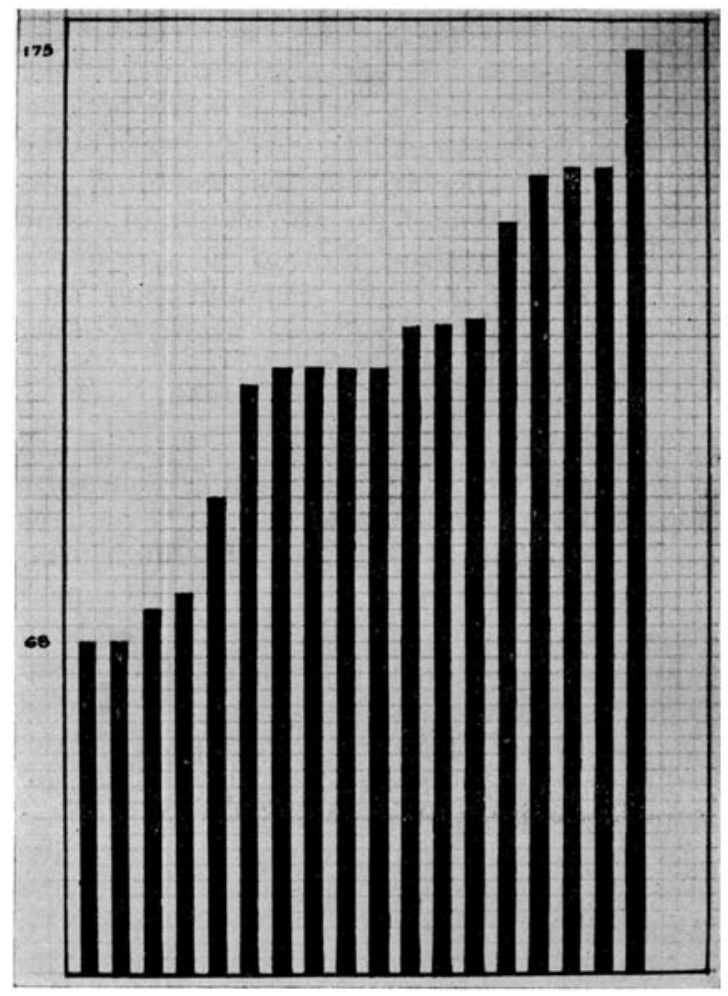

(GROUP A)

Number of perspiring pores of healthy hands

The study of healthy hands (group A) shows that there are no significant variations in the sweating rate between the right and left hands. There is not a single case of zero reading. The ring finger has a larger number of sweating pores than the little finger. It is known that different fingers have characteristically different sweating capacities and that the ring finger is usually the most active one.

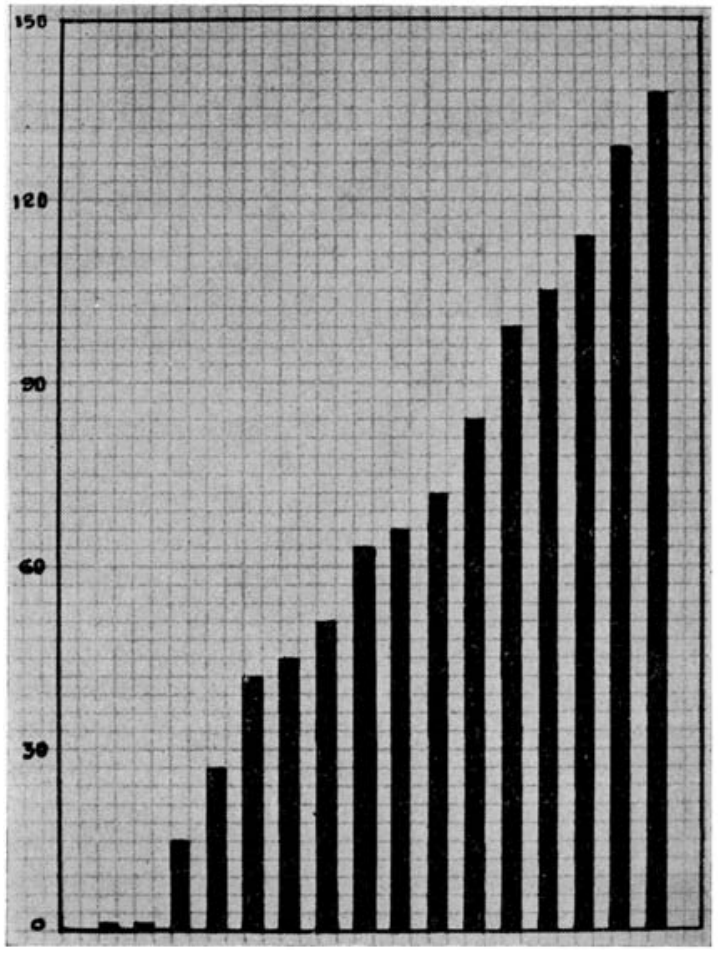

(GROUP B)

Number of perspiring pores of undeformed leprous hands

When the sweating rate of ring and little fingers of healthy hands is compared with those of undeformed hands of leprosy patients (group B) it is observed that there is a statistically significant fall in the number of sweating pores of the fingers of leprous patients even after excluding extreme cases of zero readings. The same pattern is observed when the healthy hands (group A) are compared with leprosy hands with obvious deformities (group C). Histograms I, 2 and 3 represent the readings of group A, B and C. The sweating rate of fingers of the right and left hands of leprosy patients of group B do not show any statistical difference. However in the case of group $\mathrm{C}$ though the value of $\mathrm{T}$ of sweating pores between the two hands is not significant; yet the value of $T$ is not very negligible. The number of sweating pores in the affected hands is less.

In the group B there are two cases in which both the fingers of one of the hands do not perspire at all. Both these two cases belong to maculo-anaesthetic type of leprosy. In two other 


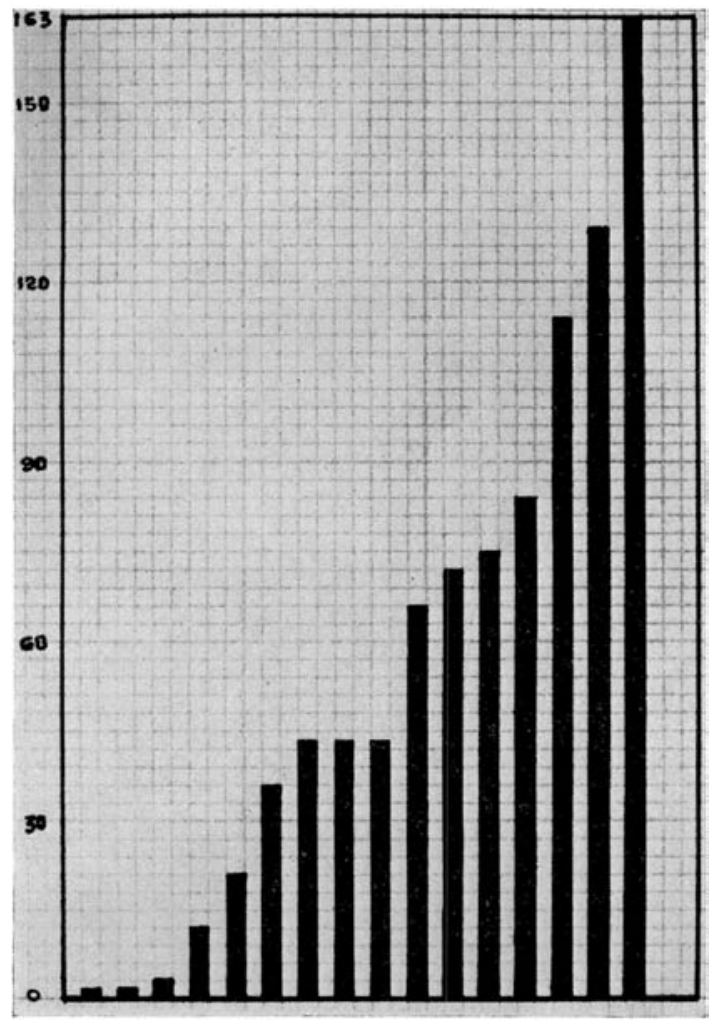

(GROUP C)

Number of perspiring pores of deformed leprous hands

cases only little fingers are affected. One of these cases is a case of maculo-aneasthetic type and the other is of lepromatous variety. In the group $\mathrm{C}$ (deformed hands) there are two cases where both the little and ring fingers of one of the hands does not show any perspiring pores. Both these hands are deformed while their counterparts are not deformed. These cases are of lepromatous type. There are five patients with zero reading of little fingers only. In this group, there is one patient each of maculo-anaesthetic neural and lepromatous types. The remaining two cases are of tuberculoid type.
OBSER VATIONS

From the present study it can be surmised that the number of sweating pores on the volar aspect of ring and little fingers of leprous hands is lesser than the normal hands. The deformities consequent on motor nerve involvement do not seem to have close co-relationship with the perspiring pores. On calculation of standard deviation it has been found out that the scattering is less in healthy hands as compared with the leprosy hands. The scattering in deformed hands of leprosy patients is more than undeformed leprosy hands.

Further studies to evaluate the individual part played by the autonomic element and the sweat glands themselves in the fall of rate of sweating pores in leprosy is under way.

\section{S UMMAR Y}

The sweating rate of the volar aspects of ring and little fingers of healthy and leprosy subjects is studied.

It is observed that there is a significant fall in the number of sweating pores in leprosy.

\section{A GKNOWLEDGEMENTS}

The authors thank

The Director, Public Health, Maharashtra State, Poona, India.

The Dean, Sassoon Hospitals, Poona, India. and

Mrs S. S. Pande for the statistical help.

\section{REFERENCES}

I Silver, A., Versaci A. and montagna, w. Studies of Sweating and sensory function in cases of peripheral nerve injuries of the hand. 7. Invest. Derm., 40, p. 243.

2 WADA, M. and TAKAGAKI, T. A simple and accurate method for detecting the secretion of sweat. Tohoku 7 . Exp. Med., 49, 284, p. I 948. 